\title{
Comportement au champ de 32 cultivars de palmier dattier vis-à-vis du bayoud : 25 années d'observations
}

\author{
M Saaidi \\ INRA Maroc, centre régional de Haouz-Présahara, BP 533, Marrakech, Maroc
}

(Reçu le 4 mars 1991; accepté le 5 mars 1992)

\begin{abstract}
Résumé - La plantation de cultivars résistants reste actuellement la seule méthode efficace de lutte contre la fusariose vasculaire du palmier dattier. La synthèse de 25 années d'observations sur des essais installés en terrain naturellement infesté à Zagora (Maroc) a permis de mieux classer les 32 cultivars testés en fonction de leur sensibilité au Bayoud. Le degré de résistance des génotypes est traduit par le taux cumulé de mortalité au bout de 25 ans après la plantation. Mais il a été également tenu compte de la présence de symptômes chroniques particulièrement fréquents sur certains génotypes et qui n'aboutissent pas obligatoirement à la mort des plantes. Sur l'ensemble, 3 cultivars se sont montrés totalement résistants (Bou Sthammi-noire, Tadment et Bou Sthammi-blanche) puisqu'ils n'ont jamais présenté de symptômes. Quatre cultivars (Sair-Layalet, Iklane, Bou Feggous-ou-Moussa et Bou-Slikhène) sont très tolérants : ils n'ont jamais montré de mortalité, même s'ils manifestent parfois quelques rares symptômes. Les autres cultivars présentent une variation continue dans leur réaction depuis une certaine tolérance jusqu'à la grande sensibilité. Cette variation continue traduit la grande diversité des génotypes et la complexité du déterminisme de la résistance. Par ailleurs, certains cultivars présentent une sensibilité limitée à leur jeune âge alors que les attaques progressent constamment sur la majorité des génotypes sensibles.
\end{abstract}

Phoenix dactylifera $=$ palmier dattier $/$ bayoud $/$ cultivar $/$ résistance au champ

Summary - Field behaviour of 32 date palm cultivars towards bayoud: a 25-year survey. Planting resistant cultivars constitutes the only efficient method available to control fusarium wilt (bayoud) in date palm. Twenty-five years ago, a trial was set up in naturally-infested open fields in Zagora (Morroco) to assess the resistance level of 32 cultivars. The observations reported in this paper enabled the cultivars to be ranked into several classes corresponding to their susceptibility to bayoud. The level of genotype resistance was characterized by the percentage of dead plants 25 years after plantation. However, in some cultivars, chronic symptoms were observed that did not necessarily induce plant mortality. Only 3 cultivars were totally resistant (Bou Sthammi-noire, Tadment and Bou Sthammi-blanche), all plants surviving without any symptom of disease. Four cultivars (Sair-Layalet, Iklane, Bou Feggous-ou-Moussa and Bou-Slikhene were tolerant to bayoud: a few plants showed symptoms of disease but did not die. Other cultivars showed a range of intermediate levels between total resistance and marked susceptibility. This continuous variation indicated a large diversity of genotypes and complexity of resistance determinism. Moreover, some cultivars were susceptible only when young, during the 10 first years of culture; in most of the cultivars however, the disease progression curve did not alter with plant age.

Phoenix dactylifera = date palm $/$ bayoud disease $/$ cultivar $/$ field resistance

\section{INTRODUCTION}

La fusariose vasculaire du palmier dattier ou bayoud ne peut être combattue actuellement de manière efficace que par l'utilisation de génotypes résistants. En effet le Fusarium oxysporum $f \mathrm{sp}$ albedinis (Malençon) $\mathrm{SN}$ et $\mathrm{H}$, agent causal de cette trachéomycose, vit et se conserve à de grandes profondeurs dans le sol (Louvet et Toutain, 1973; Djerbi, 1988; Louvet, 1991).
Depuis qu'elle a été signalée à la fin du siècle dernier au Maroc (Foex et Vayssière, 1919), cette maladie a exercé une pression de sélection sur les populations de palmier dattier, favorisant ainsi l'émergence de génotypes résistants. De tels cultivars ont été signalés en premier par Malençon (1934); ensuite Pereau Leroy (1958) entama le premier travail de sélection pour la résistance au bayoud dans la région du Ziz au Maroc. 
À partir de 1962, Louvet relança les recherches sur le Bayoud au Maroc. Une équipe franco-marocaine, animée par Toutain, réalisa d'importantes prospections de palmiers résistants (Louvet et al, 1970a). Ces premières prospections ont permis également de dresser une cartographie régionale des différents cultivars, de connaître leurs effectifs approximatifs et de repérer les plus intéressants pour leur résistance à la maladie et leur qualité de fruit (Toutain et al, 1971). L'expérimentation relative à l'évaluation de la résistance des cultivars retenus a été mise en place au domaine expérimental du Nebch à Zagora au fur et à mesure de leur repérage. Les premiers résultats ont été publiés quelques années après leur installation (Louvet et Toutain, 1973), mais la dernière mise au point remonte à plus de 10 ans (Saaïdi, 1979; Saaïdi et al, 1981).

Au terme de plus de 25 années d'observations, nous pensons utile de refaire le point sur ces essais qui constituent une expérience uni- que au monde dans ce domaine. Notre objectif est de suivre le plus longtemps possible l'évolution de la maladie sur les différents cultivars afin d'avoir une évaluation plus précise de leur degré de résistance et de connaître les modalités de l'expression de cette résistance au cours du temps.

\section{MATÉRIEL ET MÉTHODES}

\section{Choix du matériel végétal}

Cette expérimentation renferme au total 32 cultivars; 28 proviennent des principales palmeraies marocaines et 4 sont originaires de Tunisie (Louvet et al, 1970b). Le choix des cultivars a été motivé soit par la présomption de résistance (OTK, BIJ, BSL, BSTN, IKL, etc) soit par la qualité des fruits (BSK, JHL, OMH, DN, etc). Dans le tableau I nous avons résumé les principales caractéristiques de ces cultivars.

Tableau I. Principales caractéristiques des cultivars testés.

Nom complet Abréviation Consistance Précocité Qunes de culture

\begin{tabular}{|c|c|c|c|c|c|}
\hline Bou-Feggous & $\mathrm{BFG}$ & molle & assez précoce & très bonne & toutes palmeraies \\
\hline Jihel & $\mathrm{JHL}$ & demi-sèche & tardive & bonne & Draa, Bani, Saghro \\
\hline Bou-Sthammi-noire & BSTN & molle & assez tardive & moyenne & Draa, Saghro \\
\hline Iklane & $\mathrm{IKL}$ & molle & très tardive & faible & Draa, Bani \\
\hline Aguellid & AGL & demi-molle & très précoce & bonne & Draa, Saghro \\
\hline Mekt & MKT & molle & de saison & moyenne & Draa, Bani \\
\hline Tadment & TDMT & demi-sèche & précoce & bonne & Draa, Saghro \\
\hline Mest-Ali & MST & demi-molle & de saison & très bonne & Draa \\
\hline Oum-N'hale & $\mathrm{OMH}$ & molle & de saison & très bonne & Draa \\
\hline Aïssa-Youb & AIB & demi-sèche & de saison & moyenne & Draa \\
\hline Mah-Elbaïd & MLB & molle & très tardive & bonne & Draa \\
\hline Bou Skri & BSK & sèche & de saison & très bonne & Draa-Bani \\
\hline Bou Slikhène & BSL & demi-sèche & de saison & moyenne & Tafilalet \\
\hline Race-Lahmar & $R L M$ & demi-sèche & de saison & moyenne & Tafilalet \\
\hline Azegzao & $\mathrm{AZO}$ & sèche & précoce & faible & Tafilalet \\
\hline Boud-Cerdonne & $\mathrm{BCD}$ & demi-sèche & de saison & moyenne & Tafilalet \\
\hline Hals & HFS & demi-molle & de saison & moyenne & Tafilalet-oriental \\
\hline Bel-Hazit & $\mathrm{BHZ}$ & demi-molle & de saison & moyenne & Tafilalet \\
\hline Haoua & $\mathrm{HOA}$ & demi-sèche & de saison & moyenne & Tafilalet \\
\hline Admou & ADM & demi-sèche & de saison & moyenne & Tafilalet, Draa \\
\hline Bout Ittob & $\mathrm{BIT}$ & demi-sèche & de saison & bonne & Bani (Tata) \\
\hline Saïr Layalet & SLY & demi-sèche & de saison & bonne & Bani (Tata) \\
\hline Bou-Beggous-ou-Moussa & BFGM & molle & de saison & faible & Bani (Foum-Zguid) \\
\hline Bou-Sthammi-blanche & BSTB & molle & de saison & moyenne & Bani (Foum-Zguid) \\
\hline Bou-Temda & BTD & demi-sèche & de saison & moyenne & Bani \\
\hline Bou-Zeggar & BZD & demi-molle & de saison & bonne & Ghéris, Draa \\
\hline Outoukdim & OTK & demi-sèche & de saison & bonne & Todra \\
\hline Bou-ljjou & BlJ & demi-sèche & de saison & bonne & Zousfana, Gun \\
\hline Deglet-Nour (Djerid) & DNA & demi-molle & tardive & très bonne & Djerid-Tunisie \\
\hline Deglet-Nour (Kbelli) & DNB & demi-molle & tardive & très bonne & Kbelli-Tunisie \\
\hline Ftimi & FTM & demi-sèche & de saison & bonne & Djerid-Tunisie \\
\hline Oukhouet-Ftimi & OFTM & demi-sèche & de saison & bonne & Djerid-Tunisie \\
\hline
\end{tabular}




\section{Conditions expérimentales}

\section{Terrain d'expérimentation}

II s'agit de parcelles ayant déjà porté une première plantation de palmiers appartenant à un cultivar très sensible (Bou-Feggous), installée en 1944 et détruite progressivement entre 1956 et 1964 (date du début de l'installation de cette expérience). Le sol est donc naturellement infesté de Fusarium oxysporum f $\mathrm{sp}$ albedinis que nous appellerons Foa pour simplifier.

\section{Dispositif expérimental}

Un dispositif en blocs incomplets balancés a été adopté. II comporte 7 blocs identiques d'une superficie totale de 7 ha comprenant chacun 4 cultivars à tester et un témoin résistant $(\mathrm{IKL})$, représentés chacun par 120 rejets répartis en 8 répétitions de 15 plants (Bulit et al, 1967). Les arbres sont très rapprochés : $3,3 \times 3,3 \mathrm{~m}$ alors que la plantation normale est de $10 \times 10 \mathrm{~m}$, ceci pour permettre une contamination de proche en proche. En outre, un témoin sensible (BFG) figure toutes les 3 lignes. Ainsi chaque ligne de cultivar à tester est directement voisine d'une ligne BFG; ce témoin sensible est donc représenté par 21 répétitions de 15 plants soit 315 plants au total (tableau 11). Cette utilisation d'un grand nombre d'arbres sensibles répartis régulièrement dans tous les blocs permet d'estimer l'homogénéité de l'infestation du terrain et devrait maintenir un potentiel infectieux élevé et homogène.

\section{Déroulement de l'essai}

\section{Mise en place des plantations}

Les différents cultivars proviennent des palmeraies éloignées parfois de plusieurs centaines de km. Avant de pouvoir collecter et planter les rejets d'un cultivar, généralement au printemps, des prospections préalables sont nécessaires au moment de la production de dattes (automne) pour identifier les pieds mères et dénombrer les rejets plantables qu'ils portent. Pour ces raisons, la mise en place de cette expérimentation a été échelonnée sur plusieurs années. Ainsi, les cultivars marocains ont été mis en place entre 1964 et 1966 , hormis BFGM et BSTB qui ont été introduits beaucoup plus tard, respectivement en 1969 et 1972 . Quatre cultivars tunisiens ont été plantés en 1968. Le tableau III indique les cultivars plantés dans chaque bloc et les dates de mise en place.

\section{Entretien cultural}

Pour garantir la reprise végétative, tous les blocs ont bénéficié, pendant les premières années, d'un bon entretien et d'irrigations rapprochées. Ensuite, ils ont été mis progressivement dans des conditions très proches de celles qui prédominent dans la majorité des palmeraies cultivées : une culture de céréales y est pratiquée avec 6-8 irrigations d'octobre à mars; pendant le reste de l'année, quelques arrosages d'appoint sont effectués selon les disponibilités en eau.

Tableau II. Modèle de dispositif d'un bloc d'essai.

\begin{tabular}{|c|c|c|c|c|c|c|c|c|c|c|c|c|c|c|c|}
\hline $\begin{array}{l}\quad N^{\circ} \text { palmier } \\
N^{\circ} \text { ligne }\end{array}$ & 1 & 2 & 3 & 4 & 5 & 6 & 7 & 8 & 9 & 10 & 11 & 12 & 13 & 14 & 15 \\
\hline 1 & + & + & + & + & + & + & + & + & + & + & + & + & + & + & + \\
\hline 2 & . & . & . & . & . & . & . & . & . & . & . & . & . & . & . \\
\hline 3 & . & . & - & . & . & . & . & . & . & . & . & . & . & . & . \\
\hline 4 & + & + & + & + & + & + & + & + & + & + & + & + & + & + & + \\
\hline 5 & 0 & 0 & 0 & 0 & 0 & 0 & 0 & 0 & 0 & 0 & 0 & 0 & 0 & 0 & 0 \\
\hline 6 & . & . & . & . & . & . & . & . & . & . & . & • & . & . & . \\
\hline 7 & + & + & + & + & + & + & + & + & + & + & + & + & + & + & + \\
\hline 8 & . & . & . & . & . & . & . & . & . & . & . & . & . & . & . \\
\hline 10 & . & . & . & . & . & . & . & . & . & . & . & . & . & . & . \\
\hline 11 & + & + & + & + & + & + & + & + & + & + & + & + & + & + & + \\
\hline $\begin{array}{l}12 \\
" \\
" \\
" \\
\text { " }\end{array}$ & . & . & . & . & . & . & . & . & . & . & . & . & . & . & . \\
\hline 61 & + & + & + & + & + & + & + & + & + & + & + & + & + & + & + \\
\hline
\end{tabular}

$+=$ témoin sensible (Bou-Feggous); . = cultivar à tester; o = témoin résistant (IKL). 


\section{Notations et suivi}

\section{Contrôle des attaques du bayoud}

Des notations phytosanitaires ont été effectuées régulièrement tous les 6 mois pendant les 15 premières années; elles ont été ensuite moins fréquentes du fait de l'évolution plus lente de l'épidémie. Des plans précis, établis pour chaque bloc sitôt sa mise en place, permettent de situer l'emplacement des arbres sur le terrain et de suivre l'évolution des symptômes sur les sujets atteints. À la suite de chaque notation, des isolements microbiologiques ont été réalisés au laboratoire à partir de rachis de palmes présentant des symptômes pour s'assurer que l'agent responsable de l'attaque observée est bien le Foa.

\section{Notation de la vigueur des palmiers}

Afin d'évaluer l'incidence de la maladie sur le développement des palmiers survivants, nous avons noté leur vigueur végétative en 1990, en utilisant une échelle simplifiée qui les répartit en 3 catégories (tableau IV) :

\section{Palmiers vigoureux}

Les arbres sont bien développés, très productifs, hauteur de stipe 1,5 m-4 m, 40-50 palmes.

\section{Palmiers moyens}

Les arbres sont peu développés, production moyenne à faible, hauteur du stipe de $0,5-1,5 \mathrm{~m}, 30-40$ palmes.

\section{Palmiers faibles}

Les arbres sont chétifs, généralement non productifs, le stipe ne dépassant pas $50 \mathrm{~cm}$ et moins de 30 palmes.

La notation de vigueur a porté sur tous les survivants, sains ou malades (présentant des symptômes en 1990 ou apparemment guéris après une attaque antérieure).

\section{Incidents survenus au cours d'essai}

Malgré tous les soins et les précautions prises, nous n'avons pu éviter quelques aléas dus, d'une part à la biologie de la plante et à l'austérité de son milieu et d'autre part à la très longue durée de l'expérimentation. La reprise végétative des rejets est difficile à maîtriser; elle dépend du cultivar, de la qualité du rejet et des conditions de sevrage et de plantation (Toutain, 1972; Saaïdi et al, 1975). Par conséquent, bien qu'à l'origine le même nombre de rejets ait été planté par répétition (15) et par cultivar (120), le nombre de palmiers repris est plus faible (tableau III). En outre, 2 blocs $\mathrm{F}$ et $\mathrm{G}$ ont été amputés de moitié en 1981 pour d'autres expérimentations. Dans ce cas, les résultats des notations de 1990 concernent uniquement 4 répétitions au lieu de 8 . Nous devons donc, lors de l'interprétation des données, tenir compte de ces incidents qui restent en tout cas inévitables sur des essais d'une aussi longue durée, mais qui n'affectent pas la valeur des résultats.

\section{Analyse statistique des données}

Le traitement des données a été effectué à l'aide du logiciel MODLI, mis au point au laboratoire de biométrie de I'INRA à Versailles. II s'agit d'une analyse de variance non orthogonale (puisque le nombre d'arbres par répétition n'est pas constant), à 2 facteurs : le facteur bloc (les cultivars ne se trouvent pas tous dans tous les blocs), et le facteur cultivar. II a d'abord été procédé à la détection de l'effet bloc grâce aux données fournies par le témoin sensible commun aux 7 blocs, puis cet effet a été éliminé; autrement dit, pour comparer 2 cultivars se trouvant dans des blocs différents, on passe par la comparaison au témoin. Le logiciel permet de corriger les données en fonction du nombre d'arbres par répétition. Pour l'analyse, les taux d'attaque ont été transformés en $100 \times$ arcsin $\sqrt{\%}$, mais, pour une meilleure compréhension, nous présenterons aussi les résultats en pourcentages sur les tableaux et figures.

\section{RÉSULTATS}

\section{Comparaison du potentiel infectieux des blocs (fig 1)}

Le témoin sensible BFG présente la particularité de montrer une évolution rapide des symptômes maladifs, qui aboutit rapidement à la mort des

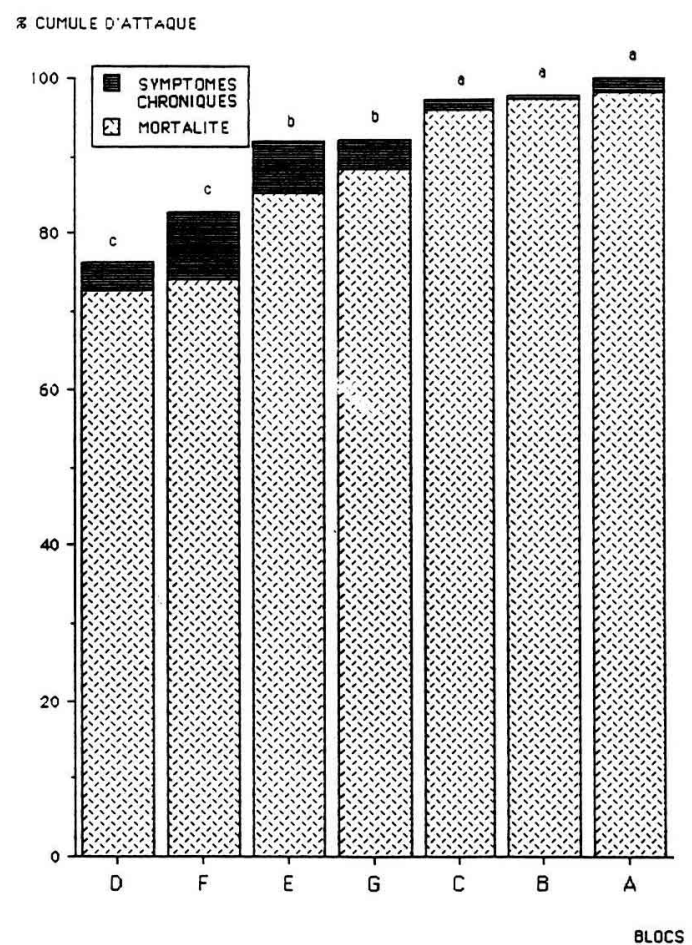

Fig 1. Mortalité due au bayoud sur le témoin sensible BFS selon les blocs. 
Tableau III. Attaques du bayoud sur les cultivars répartis dans les blocs.

\begin{tabular}{|c|c|c|c|c|c|c|c|}
\hline \multirow[t]{2}{*}{ Bloc } & \multirow[t]{2}{*}{ Cultivar } & \multirow{2}{*}{$\begin{array}{r}\text { Date de } \\
\text { plantation }\end{array}$} & \multirow{2}{*}{\multicolumn{2}{|c|}{$\begin{array}{l}\text { Palmiers repris } \\
\text { Nombre (\%) }{ }^{1}\end{array}$}} & \multicolumn{3}{|c|}{ Attaque du bayoud (\%) ${ }^{2}$} \\
\hline & & & & & Morts & Malades & Totaux \\
\hline \multirow[t]{6}{*}{$A$} & $\mathrm{BFG}^{3}$ & 1964 & 266 & 84,44 & 98,12 & 1,88 & $100 \%$ \\
\hline & $\mathrm{IKL} 4$ & " & 109 & +5 & 0 & 0 & 0 \\
\hline & BSTN & $"$ & 94 & + & 0 & 0 & 0 \\
\hline & TDMT & $"$ & 117 & + & 0 & 0 & 0 \\
\hline & AGL & $"$ & 75 & 62,50 & 25,33 & 56 & 81,33 \\
\hline & OTK & $"$ & 66 & 55,00 & 22,73 & 27,27 & 50,00 \\
\hline \multirow[t]{6}{*}{$\mathrm{B}$} & $\mathrm{BFG}$ & 1964 & 259 & 82,22 & 97,29 & 0,77 & 98,06 \\
\hline & $\mathbb{I K L}$ & $"$ & 66 & + & 0 & 0 & 0 \\
\hline & BSK & $"$ & 94 & 78,33 & 97,87 & 1,06 & 98,33 \\
\hline & $\mathrm{JHL}$ & $"$ & 75 & 62,50 & 33,33 & 17,33 & 50,66 \\
\hline & $A Z O$ & $"$ & 80 & 66,66 & 12,50 & 13,75 & 26,25 \\
\hline & BSL & $"$ & 112 & + & 0 & 13,39 & 13,39 \\
\hline \multirow[t]{7}{*}{ C } & BFG & 1964 & 268 & 85,07 & 95,89 & 1,49 & 97,38 \\
\hline & $\mathrm{IKL}$ & $"$ & 70 & + & 0 & 1,42 & 1,42 \\
\hline & HFS & $"$ & 41 & 34,16 & 85,36 & 2,43 & 87,80 \\
\hline & $B C D$ & $"$ & 78 & 65 & 14,10 & 11,53 & 25,64 \\
\hline & RLM & $"$ & 96 & 80 & 8,33 & 16,66 & 25 \\
\hline & $\mathrm{BHZ}$ & $"$ & 92 & 76,66 & 4,34 & 17,39 & 21,71 \\
\hline & BSTB & 1972 & 43 & & 0 & 0 & 0 \\
\hline \multirow[t]{7}{*}{$\mathrm{D}$} & $\mathrm{BFG}$ & 1964 & 216 & 68,57 & 72,68 & 3,25 & 75,92 \\
\hline & IKL & " & 112 & + & 0 & 0 & 0 \\
\hline & BTD & $"$ & 37 & 30,83 & 13,51 & 16,21 & 29,72 \\
\hline & BIT & $"$ & 72 & 60 & 20,83 & 5,55 & 26,38 \\
\hline & SLY & " & 85 & + & 0 & 0 & 0 \\
\hline & MKT & $"$ & 76 & 63,33 & 27,63 & 30,26 & 57,89 \\
\hline & BFGM & 1969 & 59 & + & 0 & 0 & 0 \\
\hline \multirow[t]{6}{*}{$E$} & $\mathrm{BFG}$ & 1965 & 236 & 74,92 & 85,16 & 6,77 & 91,94 \\
\hline & $\mathrm{IKL}$ & " & 54 & 0 & 0 & 0 & 0 \\
\hline & HOA & $"$ & 67 & 99,83 & 46,26 & 11,91 & 58,20 \\
\hline & BIJ & $"$ & 101 & 84,16 & 12,85 & 35,64 & 48,51 \\
\hline & ADM & $"$ & 66 & 55 & 31,81 & 30,3 & 34,84 \\
\hline & $\mathrm{BZG}$ & $"$ & 31 & 25,83 & 16,12 & 0 & 16,12 \\
\hline \multirow[t]{6}{*}{$F$} & $\mathrm{BFG}$ & 1966 & 181 & 57,46 & 74,03 & 8,83 & 82,87 \\
\hline & IKL & $"$ & 73 & + & 0 & 0 & 0 \\
\hline & MST & $"$ & 59 & 49,16 & 25,42 & 11,86 & 37,28 \\
\hline & $\mathrm{OMH}$ & $"$ & 55 & 45,83 & 20,00 & 12,72 & 72,72 \\
\hline & AIB & $"$ & 38 & 31,66 & 13,15 & 2,63 & 15,78 \\
\hline & MLB & $"$ & 54 & 45 & 1,85 & 3,70 & 5,55 \\
\hline \multirow[t]{6}{*}{$G$} & BFG & 1968 & 154 & 48,88 & 90,25 & 2,59 & 92,85 \\
\hline & $\mathrm{IKL}$ & " & 90 & + & 0 & 0 & 0 \\
\hline & DNA & $"$ & 53 & 44,16 & 69,81 & 16,98 & 86,79 \\
\hline & DNB & $"$ & 74 & 61,66 & 64,86 & 9,45 & 74,32 \\
\hline & FTM & $"$ & 78 & 65 & 16,66 & 8,97 & 25,64 \\
\hline & OFTM & $"$ & 75 & 62,50 & 10,66 & 4 & 14,66 \\
\hline
\end{tabular}

$1 \%$ calculé par rapport au nombre de rejets plantés qui est de 315 pour BFG et 120 pour les autres. $2 \%$ calculé par rapport au nombre de palmiers repris; ${ }^{3} \mathrm{BFG}=$ témoin sensible répété dans tous les blocs; ${ }^{4} \mathrm{IKL}$ témoin résistant dans tous les blocs; ${ }^{5}$ des remplacements ont été effectués sur les cultivars marqués + , on ne peut donc pas calculer le \% de reprise. 
palmiers. Les arbres vivants porteurs de symptômes disparaissent donc rapidement, ce qui explique le faible pourcentage de ce type d'arbres au moment de la dernière notation (1990). Nous verrons par conséquent que le classement des blocs ne varie pas beaucoup selon que l'on considère les pourcentages de mortalité ou les pourcentages d'attaque totale (mortalité et symptômes). Sur la figure 1, nous constatons que les 7 blocs peuvent être divisés en 3 groupes significativement différents au seuil de $5 \%$ :

- A, B, C : présentant respectivement : 98,2, 97,3 et $95,9 \%$ de mortalité;

- E, G : présentant respectivement : 90,3 et $85,2 \%$ de mortalité;

- F, D : présentant respectivement : 74 et $72,7 \%$ de mortalité.

II faut remarquer que la différence entre les blocs $C$ et $G$ est faiblement significative par suite du manque d'effectif dans ce dernier bloc, signalé précédemment.

D'une manière générale, le potentiel infectieux des parcelles expérimentales est donc élevé puisque l'on arrive à un taux moyen de mortalité de $88,7 \%$ sur un grand effectif de BFG (1580 palmiers repris sur l'ensemble des blocs). Le pourcentage moyen d'attaque est encore plus élevé $(92 \%)$. L'effet bloc étant évalué, le logiciel nous permet d'en tenir compte automatiquement lors des comparaisons des attaques sur les cultivars en y apportant un coefficient de correction.

\section{Évaluation de la résistance des cultivars}

La maladie peut se manifester de différentes façons, selon les cultivars, et parfois même, selon les arbres d'un même cultivar. Dans certains cas la mort intervient peut de temps après l'extériorisation des symptômes (quelques semaines à quelques mois), dans d'autres cas, les palmiers peuvent rester malades pendant parfois plus de 10 ans, ou même présenter des alternances de symptômes maladifs et de rémissions. Pour l'évaluation de la résistance des cultivars, il y a donc lieu de tenir compte de ces phénomènes.

D'après la figure 2 , nous remarquons que le taux de mortalité présente une variation continue d'un cultivar à l'autre, allant de la résistance totale (aucune attaque) à la grande sensibilité (98\% d'arbres morts).

Les 6 cultivars que nous avons déjà signalés comme totalement résistants (Saaïdi, 1979), le sont toujours, ce mode de classement tenant compte uniquement des mortalités permet de leur ajouter le cultivar BSL, considéré d'ailleurs traditionnellement comme résistant dans son aire de culture (Tafilalet). Les groupes homogènes que nous pouvons constituer à la sortie de l'analyse de la variance au seuil de $1 \%$ ne présentent pas des limites très nettes entre eux (fig 3 ). On peut toutefois relever que 2 cultivars très peu attaqués ( $B H Z$ et $R L M)$ ne sont pas significativement différents des 7 génotypes ne présentant aucune mortalité.

Si nous considérons les pourcentages d'attaque totale, c'est-à-dire si nous cumulons les arbres morts et les arbres vivants avec des symptômes chroniques, le classement des cultivars est nettement modifié (fig 2, tableau III). Parmi les 7 cultivars ne présentant aucune mortalité, deux manifestent des symptômes : BSL (plus de 15\%) et IKL (moins de 1\%). II faut signaler également des attaques constatées en dehors de ces essais sur 2 autres génotypes de ce groupe; il s'agit de BFGM sur lequel nous avons remarqué des symptômes sur 3 arbres dans un autre essai du domaine expérimental de Zagora et de Sly dont notre collègue Elfilali nous a rapporté de Tata des palmes atteintes. II subsiste actuellement 3 cultivars qui ne présentent aucune attaque de bayoud: BSTN, TDMT et BSTB, alors qu'il y en avait 6 il y a 10 ans (Saaïdi, 1979). Mais les symptômes constatés sur les 3 autres restent rares et n'ont jamais évolué, jusqu'à présent, au point de provoquer la mort des arbres. Pour certains cultivars, le classement est complètement modifié, ils présentent plus

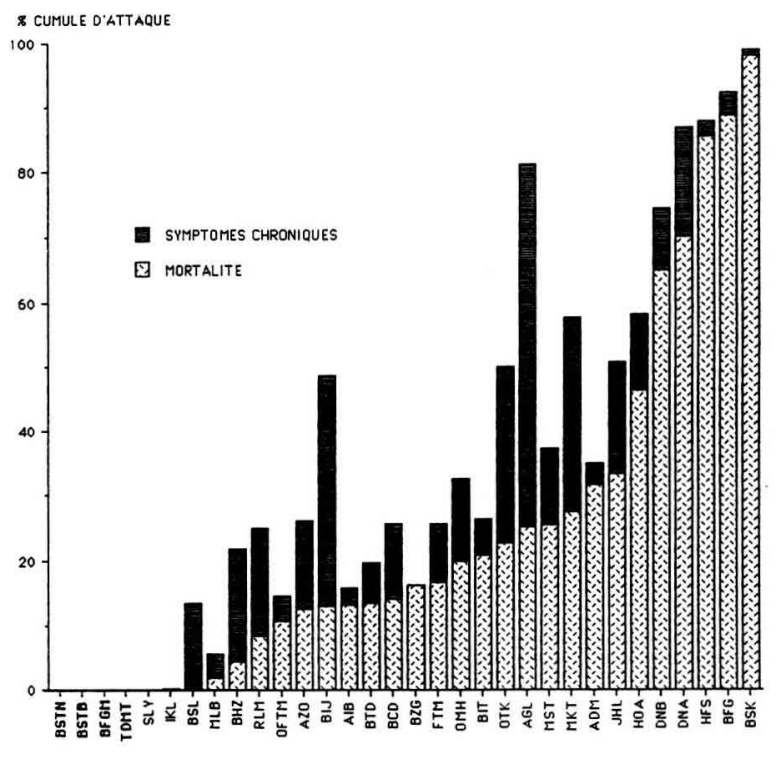

Fig 2. Classement des cultivars de palmier dattier en fonction des attaques de bayoud au bout de 25 années. 


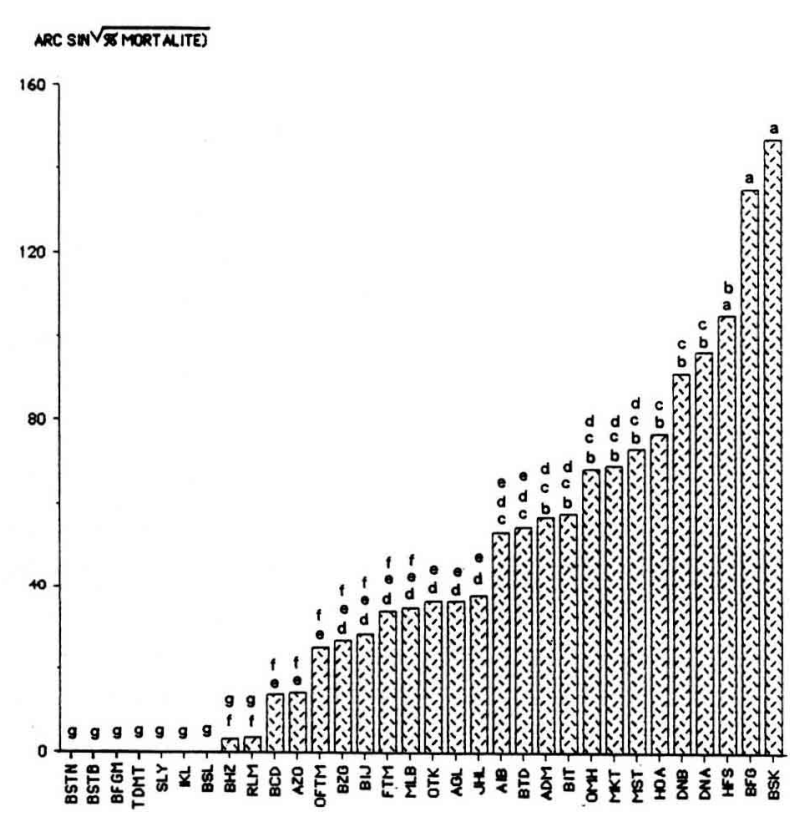

CULTNARS

Fig 3. Classement des cultivars de palmier dattier en fonction des mortalités dues au bayoud au bout de 25 années (résultats exprimés en arc sin $\sqrt{\%}$ mortalité).

d'arbres avec symptômes que d'arbres morts (AGL, BIJ, MKT, OTK); considérés de cette façon, ils sont parmi les génotypes les plus sensibles.

La comparaison de ces 2 méthodes de classement nous montre que la considération du seul pourcentage de mortalité, même si elle permet d'avoir une certaine évaluation de la résistance, ne traduit pas à elle seule toute l'incidence de la maladie sur la culture. II faut tenir compte également des attaques de Foa qui, sans aboutir à la mort de l'arbre, peuvent affaiblir et réduire sa productivité. Le problème est plus compliqué dans le cas, difficilement contrôlable, de palmiers qui peuvent héberger le parasite sans en extérioriser les symptômes classiques de dessèchement. Nous avons déjà observé ce phénomène lors de la dissection de quelques palmiers où quand nous prélevons des rejets sur des pieds mères apparemment sains, les symptômes internes de bayoud sont parfois visibles sur la plaie de coupe du rejet. Comme nous ne pouvons pour le moment détruire tous les survivants de cette expérimentation afin d'examiner leurs tissus internes, nous utiliserons une méthode indirecte d'évaluation de l'incidence de la maladie par la notation de leur vigueur. Auparavant, nous allons d'abord examiner comment les attaques du bayoud ont évolué pendant un quart de siècle sur les différents cultivars.

\section{Évolution des attaques du bayoud au cours du temps}

Pour suivre l'évolution des attaques de bayoud au cours du temps, nous avons considéré les pourcentages d'attaque totale, à 3 dates caractéristiques pour tous les cultivars (fig 4) : au jeune âge ( 5 ans), à l'entrée en production (10 ans) et à l'âge adulte (25 ans). Ensuite nous avons tracé des courbes plus détaillées d'évolution, année par année, pour 5 cultivars présentant des degrés très variables de résistance (fig $5)$.

Sur la figure 4, nous pouvons constater que le classement des cultivars change avec l'âge. Plusieurs remarques se dégagent :

- certains cultivars semblent présenter une sensibilité plus grande pendant leur jeune âge (510 ans) : MLB, BIT, OFTM, ADM, BZG et AZO;

- d'autres, par contre, sont moins attaqués quand ils sont jeunes mais ils deviennent plus sensibles avec l'âge : OTK, FTM, AIB et BHZ;

- une autre catégorie présente des attaques qui progressent régulièrement de 5 ans à 10 ans et à 25 ans : DNA, AGL, JHL, DNB et BSK.

Ces différentes réactions seraient dues, à notre avis, plus à la grande variabilité génétique de la plante qu'à la variation des conditions de milieu, certes difficiles à maintenir constantes au cours du temps pendant un quart de siècle. L'évolution constante des attaques sur les cultivars les plus sensibles confirme que les réac-

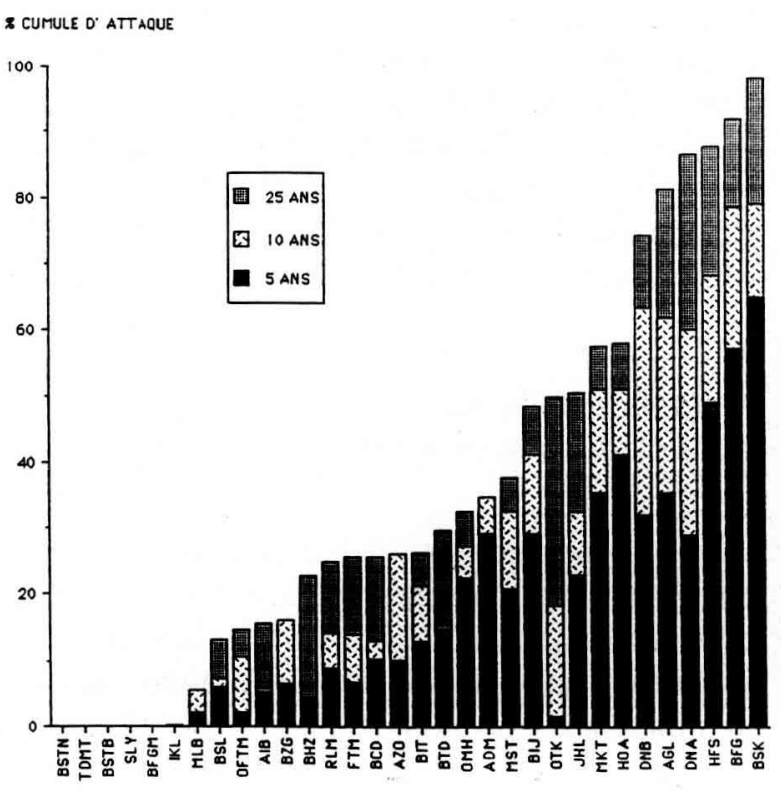

CULTIVARS

Fig 4. Réaction des cultivars de palmier dattier vis-à-vis du bayoud au cours du temps. 


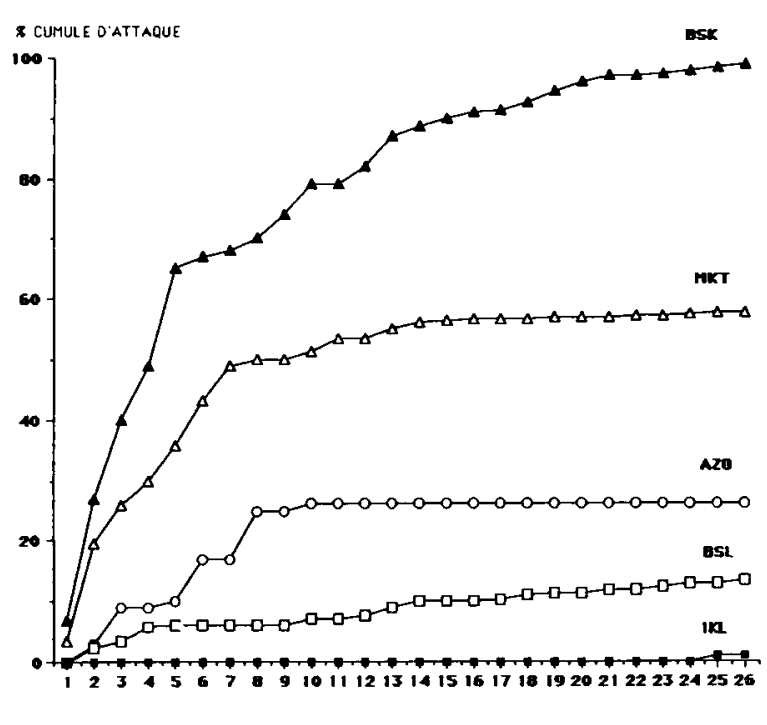

AGE EN ANNEES

Fig 5. Évolution des attaques de bayoud selon la sensibilité des cultivars.

tions des autres cultivars traduisent bien des caractères génétiques propres. La figure 5 permet de mieux suivre cette évolution. Les courbes présentent une pente d'autant plus forte que le cultivar est plus sensible (BSK). Au-delà de 10 ans, les courbes accusent toutes un palier plus ou moins marqués, sauf pour BSK. Les reprises des attaques après plusieurs années d'arrêt montrent que l'on ne peut jamais affirmer que le taux d'attaque définitif est atteint. L'allure de ces courbes pourrait également signifier que le bayoud passe par une succession de phases épidémiques et endémiques, ce qui nous semble très probable d'après nos observations sur les palmeraies contaminées et d'après les premières études de l'épidémiologie de cette maladie (Malençon, 1934; Saaïdi, 1979).

\section{Incidence de la maladie sur la vigueur des arbres}

L'analyse des données n'a pas permis de déceler des différences de vigueur bien corrélées avec les degrés de résistance des cultivars, ceci à cause, d'une part de la trop grande variation des effectifs impliqués d'un génotype à l'autre et d'autre part, du fait que le témoin sensible ne présente plus suffisamment d'arbres vivants malades pour servir de comparaison. Nous nous contenterons donc de comparer les cultivars entre eux en examinant les pourcentages d'arbres de différentes vigueurs.

D'après le tableau IV, nous pouvons constater que le classement des cultivars (palmiers sains) par vigueur croissante ne suit pas leur classement par sensibilité décroissante (fig 2). Parmi les cultivars les moins vigoureux, on trouve aussi bien des très résistants ( $B S T B, B F G M$ ), des très sensibles (HFS, DNB, DNA) que des intermédiaires (AIB, BHZ).

Dans le cas des palmiers malades, les cultivars les plus sensibles sont ceux qui présentent également les plus forts taux d'arbres de faible vigueur (tableau IV). Parfois, les survivants déjà atteints sont à $100 \%$ de faible vigueur (HOA, DNB, HFS) mais il s'agit d'effectifs très faibles. Pour certains cultivars, la vigueur semble néanmoins affectée par la maladie, puisque le taux d'arbres de faible vigueur parmi les malades est beaucoup plus élevé que le taux d'arbres de faible vigueur parmi les palmiers sains : HOA ( $100 \%$ contre $39 \%$ ), AFM $(67 \%$ contre $20 \%$ ) et BTD $(67 \%$ contre $36 \%)$. Par contre, les cultivars AGL, BIJ et MKT, qui présentent les plus forts taux d'arbres vivants malades (fig 2), montrent des pourcentages d'arbres malades de faible vigueur moins élevés $(16 \%, 22 \%$ et $34 \%)$. Ceci peut s'expliquer par le fait que ces 3 génotypes présentent par ailleurs de forts taux de rémissions parmi les palmiers ayant déjà présenté des symptômes (respectivement $79 \%, 83 \%$ et $87 \%$ ); les palmiers ainsi guéris reprennent de la vigueur.

Par ailleurs, les cultivars OTK et JHL ne présentent aucun cas de faible vigueur parmi les palmiers malades.

\section{DISCUSSION ET CONCLUSION}

Les résultats de cette expérience de terrain de longue durée traduisent toute la complexité du phénomène d'interaction entre le palmier dattier et le Foa et pourraient traduire une grande variabilité dans les réactions de défense de cette plante, ce qui engendre des degrés de résistance très variables.

Mais ce phénomène ne semble pas spécifique au palmier dattier. Gupta et Dubey (1987) l'ont signalé sur une collection de différentes origines de roquette (Eruca sativa $L$ ) soumise à un test au champ de résistance au Fusarium oxysporum. Ces auteurs ont adopté un classement très similaire de celui que nous avons déjà utilisé 
Tableau IV. Classement des survivants selon leur vigueur végétative.

\begin{tabular}{|c|c|c|c|c|c|c|c|c|c|}
\hline \multirow{3}{*}{ Cultivar } & \multicolumn{3}{|c|}{ Palmiers sains } & & \multicolumn{5}{|c|}{ Palmiers malades } \\
\hline & \multirow[t]{2}{*}{ Nombre } & \multicolumn{3}{|c|}{ Par catégorie (\%) } & \multirow[t]{2}{*}{ Cultivar } & \multirow[t]{2}{*}{ Nombre } & \multicolumn{3}{|c|}{ Par catégorie (\%) } \\
\hline & & Faibles & Moyens & Vigoureux & & & Faibles & Moyens & Vigoureux \\
\hline BTSB 1 & 38 & 79 & 21 & 0 & HOA & 9 & 100 & 0 & 0 \\
\hline HFS & 4 & 75 & 25 & 0 & DNB & 4 & 100 & 0 & 0 \\
\hline DNB & 7 & 71 & 29 & 0 & HFS & 2 & 100 & 0 & 0 \\
\hline BFGM 1 & 60 & 60 & 30 & 10 & $\mathrm{BFG}$ & 32 & 75 & 19 & 6 \\
\hline DNA & 5 & 60 & 20 & 20 & BTO & 6 & 67 & 17 & 16 \\
\hline AGL & 13 & 54 & 46 & 0 & ADM & 3 & 67 & 33 & 0 \\
\hline AlB & 8 & 50 & 38 & 12 & DNA & 5 & 60 & 20 & 20 \\
\hline SLY 1 & 87 & 48 & 28 & 24 & $\mathrm{BHZ}$ & 18 & 50 & 39 & 11 \\
\hline $\mathrm{BHZ}$ & 64 & 41 & 48 & 11 & $\mathrm{BCD}$ & 12 & 50 & 33 & 17 \\
\hline BSL 1 & 80 & 40 & 41 & 19 & $\mathrm{BIT}$ & 4 & 50 & 25 & 25 \\
\hline BCD & 55 & 40 & 46 & 14 & BSL & 15 & 40 & 33 & 27 \\
\hline BFG & 91 & 51 & 33 & 16 & MKT 2 & 21 & 34 & 38 & 28 \\
\hline HOA & 28 & 39 & 47 & 14 & RLM & 16 & 25 & 50 & 25 \\
\hline BIJ & 51 & 39 & 35 & 26 & $\mathrm{BIJ} 2$ & 36 & 22 & 53 & 25 \\
\hline BSTN 1 & 85 & 38 & 21 & 41 & AGL 2 & 44 & 16 & 39 & 45 \\
\hline BTD & 33 & 36 & 43 & 21 & $A Z O$ & 12 & 8 & 9 & 83 \\
\hline MKT & 31 & 32 & 42 & 26 & $\mathrm{JHL}$ & 7 & 0 & 72 & 29 \\
\hline RLM & 73 & 29 & 46 & 25 & FTM & 5 & 0 & 20 & 80 \\
\hline FTM & 34 & 27 & 32 & 41 & OTK & 29 & 0 & 41 & 59 \\
\hline TDMT 1 & 98 & 27 & 20 & 53 & MST & 2 & 0 & 100 & 0 \\
\hline JHL & 42 & 26 & 55 & 19 & $\mathrm{OMH}$ & 1 & 0 & 100 & 0 \\
\hline $\mathrm{BZG}$ & 23 & 26 & 26 & 48 & AIB & 1 & 0 & 100 & 0 \\
\hline OTK & 21 & 24 & 38 & 38 & IKL & 1 & 0 & 100 & 0 \\
\hline MST & 18 & 22 & 50 & 28 & MLB & 0 & 0 & 0 & 0 \\
\hline $\mathrm{IKL} 1$ & 407 & 20 & 30 & 50 & OFTM & 0 & 0 & 0 & 0 \\
\hline BIT & 55 & 20 & 53 & 27 & BSTN & 0 & 0 & 0 & 0 \\
\hline ADM & 46 & 20 & 37 & 43 & BSTB & 0 & 0 & 0 & 0 \\
\hline OFTM & 40 & 18 & 35 & 47 & SLY & 0 & 0 & 0 & 0 \\
\hline$A Z O$ & 59 & 17 & 22 & 61 & BFGM & 0 & 0 & 0 & 0 \\
\hline MLB & 30 & 13 & 17 & 70 & TDMT & 0 & 0 & 0 & 0 \\
\hline
\end{tabular}

${ }^{1}$ Des remplacements ont été effectués sur ces cultivars, ce qui augmente le \% des arbres de faible vigueur car plus jeunes; ${ }^{2}$ ces 3 cultivars présentent des \% élevés de cas de rémission (AGL : $75 \%$, BJ : $83 \%$ et MKT : $95 \%$ ).

pour le palmier (Saaïdi et al, 1981) : ils ont distingué des génotypes tolérants, assez tolérants, sensible et très sensibles. Mais seules les lignées tolérantes et assez tolérantes ont été retenues pour les programmes de sélection futurs. De même Renard et al (1989) ont trouvé plusieurs gammes de résistance chez le palmier à huile en inoculant les jeunes plantes en pépinière par le Fusarium oxysporum $f \mathrm{sp}$ elaeidis.

Les conditions expérimentales rencontrées à Zagora nous semblent satisfaisantes pour permettre une bonne évaluation de la résistance au champ des différents cultivars. En effet, malgré les différences constatées, le potentiel infectieux du sol reste en général élevé, puisque l'on dépasse $90 \%$ d'attaque en moyenne sur le témoin sensible. Les différences entre les blocs peuvent être attribuées à une variation des facteurs bioécologiques qui conditionnent le développement des maladies d'origine tellurique (Louvet et Alabouvette, 1988). Dans notre situation, la nature plus argileuse du sol des blocs $D$ et $F$ pourrait être responsable de leur taux d'attaque relativement faible. Visant avant tout un objectif de sélection, nous ne nous sommes pas attachés à élucider ces phénomènes, sur lesquels d'ailleurs plusieurs autres chercheurs travaillent actuellement (Sedra et Rouxel, 1989; Amir et al, 1989; Oihabi et al, 1990).

Toutefois ce terrain, avec son hétérogénéité, reflète assez bien ce qui se passe dans les sols des palmeraies. II permet donc de juger la résistance au champ des cultivars et de les comparer entre eux, ce qui est le plus important. 
Mais l'évaluation comparative de la résistance des génotypes pose d'autres problèmes liés aux divers modes et stades de son expression. Certes, le classement basé sur les seuls taux de mortalité traduit l'effet le plus marquant de la maladie, mais il reste incomplet car il n'englobe pas toute l'incidence de la maladie sur la culture. La prise en considération des arbres présentant des symptômes, mais restés vivants, est également importante car ce phénomène caractérise certains cultivars ( $A G L, B I J$ et MKT). Le génotype BSL peut être classé comme non attaqué si l'on considère uniquement les mortalités, alors qu'il présente $13 \%$ d'arbres avec symptômes.

Les cultivars ayant montré une sensibilité faible et/ou limitée au jeune âge ne doivent pas être négligés, surtout quand ils présentent une très bonne qualité de fruit $(\mathrm{OMH})$ ou une bonne qualité et une grande vigueur (BZG et $A D M$ ). Mais d'un autre côté, la reprise des attaques après plusieurs années d'arrêt sur certains génotypes (AIB, BZH, BTD) nous incite à la prudence et nous fait penser que les cultivars à résistance intermédiaire finiront par disparaître: ils ne peuvent donc être intéressants que si leur productivité compense largement cet inconvénient majeur. Les agriculteurs de certaines régions (Tafilalet au Maroc) sont prêts à accepter un certain taux de mortalité sur les cultivars de grande valeur marchande.

Le fait que 3 cultivars parmi les 6 que nous avions déjà classés comme totalement résistants à l'âge de 10 ans (Saaïdi, 1979) présentent à 25 ans quelques rares cas de symptômes, montre que l'on ne peut jamais être absolument sûr du maintien d'un niveau de résistance constant avec le temps. Mais ces rares attaques, qui n'ont jamais abouti à des mortalités ne compromettent pas l'intérêt de ces cultivars. II s'agit en fait d'un phénomène fréquent chez les variétés de plusieurs espèces végétales résistantes à diverses maladies (Messiaen, 1981). Les 3 cultivars qui n'ont présenté jusqu'à présent aucune attaque (BSTN, TDMT et BSTB) sont probablement très apparentés : sur le plan de la morphologie des organes végétatifs, ils se ressemblent beaucoup. La BSTN, variété très ancienne (150 ans ou plus), très abondamment représentée dans le Draa serait l'ancêtre commun des 2 autres.

Le degré de résistance d'un génotype peut donc être mesuré par le pourcentage d'arbres atteints de bayoud au bout d'un temps donné, dans des conditions données. Dans le cas des essais de plein champ, le facteur temps joue un grand rôle; le classement de nos cultivars qui varie à 5 ans, 10 ans et 25 ans le confirme (fig 4).

Pour mieux évaluer l'incidence du Bayoud sur la vigueur des palmiers survivants, il serait utile d'en sacrifier une grande partie et de les disséquer pour contrôler la présence et l'intensité des symptômes internes et tester l'agressivité des souches de Foa que l'on peut en isoler. Mais d'ores et déjà, l'effet dépressif de la maladie semble évident sur les survivants de certains cultivars (HOA, DNB et BFG). Ce phénomène est plus net dans le cas du palmier à huile attaqué par le Fusarium oxysporum $\mathrm{f} \mathrm{sp}$ elaeidis où la majorité des arbres atteints présentent des symptômes chroniques mais n'en meurent pas rapidement. Par contre, ils restent très affaiblis et peu productifs (Renard et al, 1972; Defranqueville, 1984).

Enfin, même si l'évaluation de la résistance au champ est très lente et complexe, elle restera toujours la seule méthode qui reflète la réalité du terrain et elle servira à chaque fois de référence. Avec le développement de la micropropagation in vitro, des techniques d'évaluation de la résistance plus simples et plus rapides peuvent être mises au point par inoculation artificielle des vitroplants. Des résultats ont déjà été obtenus (Sedra, 1989a) mais il reste encore à affiner ces techniques (Louvet, 1991) pour qu'elles reflètent bien la résistance réelle au champ. II faut parvenir à éliminer tous les arbres sensibles au risque de perdre quelques arbres résistants.

À l'avenir, il sera nécessaire d'approfondir les études des relations hôte-parasite pour mieux élucider les différents types de résistance constatés sur le terrain. Les recherches déjà réalisées dans ce domaine portent sur les structures anatomiques et les réactions mécaniques de la plante qui, selon les auteurs, n'ont pas de rôle important (Oihabi, 1984) ou au contraire sont impliquées dans la résistance chez certains cultivars (Belarbi, 1989). La nature biochimique de la résistance a été également étudiée : les analyses foliaires ont permis de mettre en évidence des taux de peroxydases plus élevés chez les cultivars résistants (Baaziz et Saaïdi, 1988; Baaziz, 1989). Des phyto-alexines capables d'inhiber le Foa ont été décelées à des doses plus élevées dans les racines du cultivar résistant BSTN préalablement inoculées avec le foa (Assef et al, 1986). Enfin, les exsudats racinaires des génotypes résistants semblent freiner la multiplication du Foa (Assoir et Djerbi, 1989). 
Un des principaux résultats de ce travail est la mise en évidence de 6 cultivars présentant un très bon niveau de résistance au bayoud associé, selon les critères actuels, à une faible qualité des fruits. Pour obtenir des clônes de grande valeur agricole et commerciale, il faut recombiner résistance, qualité et productivité, caractères actuellement dissociés, en croisant des génotypes complémentaires. Les 6 clônes mis en évidence pour leur résistance élevée constituent un matériel de choix pour réaliser ces croisements. Des génotypes prometteurs ont déjà été obtenus dans des croisements dirigés entre ces clônes et des mâles apportant une bonne qualité de fruits (Saaïdi, 1989, 1990). Des génotypes également intéressants ont été obtenus dans des descendances en croisements libres.

Le Maroc reste le pays le plus avancé dans ce domaine, par suite de son antériorité dans l'apparition de la maladie et des bonnes orientations de recherches définies dès le départ (Bulit et al, 1967; Louvet et al, 1970b; Toutain et Louvet, 1974). La recherche agronomique marocaine a toujours soutenu ce programme de longue haleine et en a assuré la continuité. Récemment, cette voie a été suivie en Algérie où des prospections importantes ont été entreprises (Brac de la Perrière et $a l, 1989$ ) et même en Tunisie où l'on est en train d'évaluer le patrimoine génétique phoenicicole avant l'arrivée du bayoud (Rhouma, 1989).

\section{REMERCIEMENTS}

Nous tenons à remercier :

- Jean Louvet (directeur de recherches, pathologie végétale, INRA Dijon) et Hubert Bannerot (directeur de recherches, génétique, INRA Versailles) pour l'intérêt qu'ils continuent à accorder à ce travail et pour avoir bien voulu relire notre texte et nous faire part de leurs remarques;

- André Kobilinsky (directeur de recherches, biométrie, INRA Versailles) qui nous a été d'un grand secours pour l'analyse des données.

\section{RÉFÉRENCES}

Amir H, Riba O, Amir A, Bounaga N (1989) Influence de la salinité des sols de palmeraies sur les Fusarium. I. Relations entre la densité des populations de Fusarium et la conductivité des sols. Rev Écol Biol Sol 26(4), 391-406

Assef GM, Assari K, Vincent EJ (1986) Occurrence of an antifungal principle in the root extract of a bayoud-resistant date palm cultivar. Neth $J$ Plant Pathol 92, 43-47
Assoir NM, Djerbi M (1989) Rôle des exsudats racinaires dans le système de défense du palmier dattier à l'égard du Fusarium oxysporum $f \mathrm{sp}$ albedinis, agent causal du bayoud. Sémin maghréb génét palmier dattier, 2-7 déc 1989, Adrar, Algérie, Doc FAO/INRA, Alger

Baaziz M (1989) The activity and preliminary characterization of peroxidases in leaves of cultivars of date palm, Phoenix dactylifera L. New Phytol 111, 403-411

Baaziz M, Saaïdi M (1988) Preliminary identification of date palm cultivars by esterase isoenzymes and peroxidase activities. Can J Bot 66, 89-93

Belarbi R (1989) Cytohistologie de quelques variétés de palmier dattier : relation éventuelle avec leur résistance au bayoud. Sémin maghréb génét palmier dattier, 2-7 déc 1989, Adrar, Algérie. Doc FAO/ INRA, Alger

Brac de la Perrière RA, Benkhalifa A (1989) Traitement de données de prospections et d'inventaires de la palmeraie dattière. Sémin maghréb génét palmier dattier, 2-7 déc 1989, Adrar, Algérie. Doc FAO/INRA, Alger

Bulit J, Louvet J, Bouhot, D, Toutain G (1967) Travaux sur les fusarioses. I. Travaux sur le bayoud, fusariose vasculaire du palmier dattier en Afrique du Nord. Ann Épiphyt 18(2), 213-239

Djerbi M (1988) Les maladies du palmier dattier. Projet régional de lutte contre le bayoud, Alger, FAO (RAB/84/018), $127 \mathrm{p}$

Djerbi $M$, Aouad L, Elfilali $H$, Saaïdi $M$, Allaoui $M$ (1985) Résultats préliminaires sur la recherche de "Khalts" de haute qualité dattière et résistants au Bayoud parmi les populations locales de palmier dattier. Sémin Nat Agron Saharienne, INRA/Fac Sci, Marrakech, 19-29

Foex $E$, Vayssière $P$ (1919) Les maladies du dattier au Maroc. J Agric Trop 162, 336-339

Franqueville $H$ (de) (1984) La fusariose du palmier à huile : relation entre la résistance en pépinière et la résistance au champ. Oléagineux $39,514-518$

Gupta AK, Dubey MM (1987) Field reaction of taramira (Eruca sativa L) germplasm to Fusarium wilt. Indian J Mycol Plant Pathol 17, 74

Louvet J (1991) Que devons-nous faire pour lutter contre le bayoud? In: Physiologie des arbres et arbustes en zones arides et semi-arides. Groupe d'étude de l'arbre, Paris, 337-346

Louvet J, Alabouvette C (1988) Les conditions de développement du parasitisme racinaire. Phytoma 402, 32-34

Louvet J, Toutain G (1973) Recherches sur les fusa rioses. VIII. Nouvelles observations sur la fusariose du palmier dattier et précisions concernant la lutte. Ann Phytopathol 5, 35-52

Louvet J, Bulit J, Toutain G (1970a) Le Bayoud, fusariose vasculaire du palmier dattier. Symptômes et nature de la maladie. Moyens de lutte. Al-Awamia (INRA, Maroc) 35, 161-182 
Louvet J, Bulit J, Toutain G (1970b) Comparaison de la résistance au bayoud de quatre clones tunisiens de palmier dattier. Al-Awamia (INRA, Maroc) 34, 111-118

Malençon $G$ (1934) La question du bayoud au Maroc. Ann Cryptogam Exot, Paris, 7, 1-41

Messiaen CM (1981) Les variétés résistantes. Méthode de lutte contre les maladies et ennemis des plantes. INRA, Paris, $379 p$

Oihabi A (1984) Étude comparative du comportement vis-à-vis du Fusarium oxysproum $\mathrm{f} s \mathrm{~s}$ albedinis de jeunes plantes sensibles et résistantes de palmier dattier. Thèse de $3^{e}$ cycle, Université Marrakech, Maroc

Pereau Leroy $P$ (1958) Le palmier dattier du Maroc. Ministère de l'Agriculture, Maroc, $142 p$

Renard JL, Gascon JP, Bachy A (1972) Recherches sur la fusariose du palmier à huile. Oléagineux 12 , 581-591

Renard JL, Noiret JM, Meunier RJ (1980) Sources et gammes de résistance à la fusariose chez le palmier à huile. Oléagineux 35(8-9), 388-399

Saaidi M (1979) Contribution à la lutte contre le bayoud, fusariose vasculaire du palmier dattier. Thèse Doctorat, Université Dijon, $140 \mathrm{p}$

Saaïdi M (1989) Programme d'amélioration génétique du palmier dattier au Maroc, méthodologie, résultats et problèmes. Sémin maghréb génét palmier dattier, 2-7 déc 1989, Adrar, Algérie, Doc FAO/ INRA, Alger

Saaïdi M (1990) Amélioration génétique du palmier dattier : critères de sélection, techniques et résultats. Option Méditer Sem A/n ${ }^{\circ} 11,133-153$
Saaïdi M, Duvauchelle S, Toutain G (1975) Multiplication du palmier dattier : étude de quelques facteurs conditionnant la reprise végétative des rejets de palmier dattier en plein champ. Fruits 34(9), 555561

Saaïdi M, Toutain G, Bannerot H, Louvet $J(1981)$ La sélection du palmier dattier (Phoenix dactylifera $\mathrm{L}$ ) pour la résistance au bayoud. Fruits 36(4), 214-249

Sedra My H (1989a) Recherche d'une méthode fiable d'évaluation de la résistance des vitroplants du palmier dattier : mise au point et applications. Sémin maghréb génét palmier dattier, 2-7 déc 1989, Adrar, Algérie. Doc FAO/INRA, Alger

Sedra My H (1989b) Sélection en palmeraie de palmiers dattiers résistants au Bayoud et de bonne qualité de fruits : résultats et problèmes. Sémin maghréb génét palmier dattier, 2-7 déc 1989, Adrar, Algérie, Doc FAO/INRA, Alger

Sedra My H, Rouxel F (1989) Résistance des sols aux maladies. Mise en évidence de la résistance d'un sol de la palmeraie de Marrakech aux fusarioses vasculaires. Al-Awania 66, 35-54

Toutain G (1972) Observations sur la reprise végétative des rejets de palmier dattier. Al-Awamia 43 , 113-132

Toutain G, Louvet J (1974) Lutte contre le bayoud. IV. Orientations de la lutte au Maroc. Al-Awamia 53, 141-162

Toutain G, Bachra A, Chari A (1971) Cartographie variétale de la palmeraie marocaine. Doc INRA, Maroc, $242 \mathrm{p}$ 